\title{
BMJ Open Clinical predictors of 2-year outcome of resective epilepsy surgery in adults with refractory epilepsy: a cohort study
}

\author{
B Kanchanatawan, ${ }^{1}$ C Limothai, ${ }^{2}$ T Srikijvilaikul, ${ }^{3}$ M Maes ${ }^{1,4}$
}

To cite: Kanchanatawan B Limothai C, Srikijvilaikul T, et al. Clinical predictors of 2-year outcome of resective epilepsy surgery in adults with refractory epilepsy: a cohort study. BMJ Open 2014;4:e004852.

doi:10.1136/bmjopen-2014004852

- Prepublication history for this paper is available online. To view these files please visit the journal online (http://dx.doi.org/10.1136/ bmjopen-2014-004852).

Received 14 January 2014 Revised 20 March 2014 Accepted 27 March 2014

CrossMark

\footnotetext{
${ }^{1}$ Faculty of Medicine, Department of Psychiatry, Chulalongkorn University, Bangkok, Thailand ${ }^{2}$ Faculty of Medicine, Department of Medicine, Chulalongkorn University, Bangkok, Thailand

${ }^{3}$ Department of Surgery, Prasat Neurological Institute, Bangkok, Thailand

${ }^{4}$ Department of Psychiatry, Deakin University, Geelong, Australia
}

\footnotetext{
Correspondence to Dr M Maes; dr.michaelmaes@hotmail. com
}

\begin{abstract}
Objectives: Resective epilepsy surgery is currently a standard treatment for intractable epilepsy. Seizure freedom and discontinuation of antiepileptic drugs are the ultimate goals of epilepsy treatment. This study was carried out to delineate (1) possible differences in the success rate of epilepsy surgery 6 and 24 months after surgery; and (2) the clinical predictors of a good response to surgery.
\end{abstract}

Setting: This is a cohort study performed at a tertiary care unit of a university hospital.

Participants: In this cohort study, 189 adults with intractable epilepsy who underwent epilepsy surgery were included. We collected clinical data at three time points, that is, preoperative and 6 and 24 months after surgery.

\section{Primary and secondary outcome measures:}

Engel class I-IV classification was the primary outcome measure of epilepsy surgery. The authors statistically adjusted Engel class I-IV classification for postoperative changes in antiepileptic drugs and used this new classification as a secondary outcome variable.

Results: The success rate was $78.8 \% 6$ months after surgery and increased to $88.3 \% 24$ months after surgery. This success rate was reflected not only by the reduced number of seizures postsurgery, but also by a reduced dosage and use of antiepileptic drugs. Logistic regression analysis showed that a successful outcome of surgery is predicted by having temporal rather than extratemporal lobe epilepsy and less than nine presurgery seizures per month, while a positive familial history of epilepsy, younger age and dysphoric symptoms, the first 3 months after surgery, significantly worsened the outcome of surgery. Duration of illness, age at onset, epilepsy location, type of lesions and the presence of psychosis were not significant in predicting treatment outcome.

Conclusions: These findings have clinical relevance in that a better selection of patients based on the significant clinical predictors will increase the success rate of epilepsy surgery and treatment.

\section{INTRODUCTION}

In most state-of-the-art epilepsy units, resective epilepsy surgery is currently the standard

\section{Strengths and limitations of this study}

- The authors analyse a large series $(n=189)$ of consecutively admitted patients with refractory epilepsy who underwent epilepsy surgery and delineate the differences in surgical outcome between 6 and 24 months after surgery, clinical predictors of good surgical outcome (Engel class I) and the effects of withdrawal of antiepileptic drugs (AEDs).

- This is the first study that adjusts the results of epilepsy surgery outcome data for changes in AEDs. The authors propose that Engel's classification into four classes may not be adequate because postsurgery patients allocated to an Engel class who had their AEDs discontinued or reduced differ from those belonging to the same Engel class but who had an increased or unchanged AED intake. Therefore, the authors suggest that the Engel class classification should be refined taking into account postoperative changes in AED status.

- The shorter follow-up period (24 months) is a limitation of the study. The high success rate of epilepsy surgery in this study (ie, $88.3 \%$ at 24 months) may be explained by our strict selection criteria. This cohort comprises $2 \%$ MRI-negative epilepsy and $>90 \%$ temporal lobe surgery patients, and therefore our findings cannot be readily extrapolated to more heterogeneous cohorts.

treatment for intractable epilepsy. Generally, the success rate, defined as a seizure-free status or Engel class I, is between $62 \%$ and $71 \%$, as compared to $14 \%$ in non-operated cases. ${ }^{12}$ For example, in the Epilepsy Unit of King Chulalongkorn Memorial Hospital, Bangkok, Thailand, the success rate 24 months after surgery is $66.7 \%$ as compared to $5 \%$ in cases without surgery. ${ }^{3}$ Clinical experience is that some patients with epilepsy who are non-responders to surgery in the first few months after surgery become seizure free and thus responders some months later. 
In order to improve the success rate to epilepsy surgery, selection criteria for surgery based on clinical and biological characteristics of responders and nonresponders should be delineated. Neurological predictors include type of resection, preoperative aura, presence of postoperation spikes, ${ }^{2}$ extratemporal resection, simple partial seizure, ${ }^{4}$ long seizure duration, number of seizures per month at baseline, secondarily generalised seizures and ictal dystonia. ${ }^{5}$ It is debated whether psychiatric problems may modulate the outcome to epilepsy surgery. Some studies show that preoperative psychiatric diagnoses may predict a negative outcome to epilepsy surgery. ${ }^{6-10}$ Other studies, however, report that a history of psychiatric diagnosis is not a predictor for surgery outcome. ${ }^{11}$ It has remained elusive, however, whether clinical variables, such as duration of illness, type of epilepsy, epilepsy location and a familial history of epilepsy, may predict a good outcome, and whether a combination of these and other factors may improve the prediction.

To complicate matters, discontinuation of antiepileptic drugs (AEDs) may interfere with surgery outcome. Surgery may allow to taper down or even discontinue AED intake in some patients after epilepsy surgery. On the other hand, tapering down AEDs may cause seizure recurrence in about a third of patients. ${ }^{12}$ This indicates that when assessing epilepsy surgery outcome, one has to take the AED state into account. This may also indicate that Engel's classification into four classes is not always adequate. For example, it is obvious that there is a difference between postsurgery patients allocated to an Engel class and who had their AEDs discontinued or reduced and those belonging to the same class but who had an increased or unchanged AED intake. One approach is to adjust the Engel classes for postoperative changes in AED state.

This study was carried out to delineate (1) the success rate of epilepsy surgery 6 and 24 months after surgery as assessed by means of Engel classes; and (2) clinical predictors of a good treatment response while adjusting for the effects of discontinuation or reduction of AEDs on this prediction.

\section{PATIENTS AND METHODS}

This is a cohort study performed at the Comprehensive Epilepsy Unit, King Chulalongkorn Memorial Hospital, Bangkok, Thailand. We consecutively included (from October 2005 until June 2008) all patients with intractable epilepsy who were selected for epilepsy surgery and attended the Comprehensive Epilepsy Unit for postoperative evaluations. One hundred and eighty-nine patients were included in this study. We collected data at three different time points, that is, preoperation, and 6 and 24 months after surgery. We collected sociodemographic data, age at onset, duration of illness, familial history of epilepsy, number of seizures per month before and 6 and 24 months after surgery, use of AEDs (type and dosage) before and 6 and 24 months after surgery, using semistructured interviews performed by a trained master degree research psychologist. Epilepsy-related characteristics, including epilepsy location and type of epilepsy, were rated by senior neurologists using neurological, medical and neurosurgical records, $24 \mathrm{~h}$ EEG reports and brain imaging techniques, that is, MRI. The postsurgery data at 6 and $24 \mathrm{~h}$ were used to make the Engel class diagnoses in class I (no disabling seizures), class II (almost free of seizures), class III (worthwhile improvement with $>50 \%$ reduction in disabling seizures) and class IV (no worthwhile improvement). The psychiatric Diagnostic and Statistical Manual of Mental Disorders, Fourth Edition (DSM-IV) diagnosis psychosis (before surgery) was made by a trained master degree research psychologist and a senior psychiatrist using the Mini International Neuropsychiatric Interview (MINI) in a Thai validated version. ${ }^{13}$ Dysphoric disorder was defined as an emotional response within the first 3 months after epilepsy surgery characterised by labile mood, crying spells, behavioural outbursts, sleep problems, concentration disorders and/or irritability. All participants gave written informed consent to participate.

\section{STATISTICS}

We used analyses of contingency tables $\left(\chi^{2}\right.$ tests $)$ or Fisher's exact probability test to check differences in the distribution of variables among two or more study groups. Relationships between variables were assessed using Pearson's correlation coefficients. General and generalised linear model analyses were used to predict dependent variables by means of different explanatory variables. We used analyses of variance (ANOVA) in order to ascertain differences in continuous variables between two and more study groups. Multiple post hoc differences were assessed by means of Tukey's tests. Binary logistic regression analysis was used to define the associations between a dichotomous dependent variable and a set of independent variables. We used the logistic regression coefficients of the explanatory variables in the final equation to estimate ORs with CIs. We used the sign test to assess the differences in the Engel classes (considered as ordinal scaled variables) 6 and 24 months after epilepsy surgery. The sign test is a nonparametric test statistic which can be employed to test paired samples of ordinally scaled variables and which uses only directional and not magnitude information. The effects of epilepsy surgery on the discontinuation of AEDs were analysed using the McNemar test, a nonparametric test to analyse differences in repeated measurements (RMs) of binary data. The surgery effects on the number of seizures and AED use was analysed using factorial RM design ANOVA or the Wilcoxon signed-rank test, a non-parametric test used to check differences in pairs of data. The results of parametric tests were checked using non-parametric tests including 
Table 1 Engel class classifications in 189 patients, 6 and 24 months after epilepsy surgery

\begin{tabular}{llrlll}
\hline Engel classes & \multicolumn{5}{l}{ 24 months } \\
\hline \multirow{3}{*}{6 months } & & I & II & III & IV \\
& I & 144 & 3 & 1 & 1 \\
& II & 10 & 3 & 3 & 0 \\
& III & 0 & 0 & 2 & 0 \\
IV & 13 & 3 & 2 & 4 \\
\hline
\end{tabular}

The distribution of the patients in classes I-IV is significantly different between months 6 and 24 (sign test: $z=-3.17, p=0.002$, negative differences $=28$, positive differences $=8$ and ties $=153$ ).

Spearman's rank order correlation coefficients and the Kruskal-Wallis test. Data were analysed using SPSS. There were no missing values in our data set. Statistical significance was set at $\alpha=0.05$ (two tailed).

\section{RESULTS}

\section{Characteristics of Engel classes}

Table 1 shows that there was a significant difference in Engel class distribution between the two time points. The sign test showed that there were significantly more negative differences than positive differences, indicating that some patients improved from month 6 to month 24 . For example, 6 months after surgery, 149 patients were allocated to class I, while 24 months after surgery, 167 were allocated to class I. Twenty-three patients who were allocated to Engel classes II, III or IV 6 months after surgery were reallocated to Engel class I 24 months after surgery, showing that their status had improved. We have analysed whether any of the variables listed in table 2 was associated with this subgroup of patients who had improved, but not one of the variables was significant. For example, there were no significant associations between improvement in Engel class classification and family history of epilepsy $\left(\chi^{2}=1.16, \mathrm{df}=1, \mathrm{p}=0.281\right)$, temporal lobe epilepsy (TLE) versus extratemporal lobe epilepsy (ETLE; $p=0.415$ by Fisher's exact probability test) and type of epilepsy ( $\mathrm{p}=0.599$ by Fisher's exact probability test). In order to examine possible associations between the improvement in Engel class classification and use of AEDs, we have performed RM design ANOVAs with dosage of AEDs at 6 and 24 months as a time factor and improvement in Engel class I classification as a factor. We found a significant time $\times$ group interaction only for levetiracetam dosage $(\mathrm{F}=5.47, \mathrm{df}=1 / 187$, $\mathrm{p}=0.02$ ). Logistic regression analysis with use of AEDs

Table 2 Demographic data and Engel classification 24 months after surgery in 189 patients

\begin{tabular}{|c|c|c|c|c|c|c|c|}
\hline Variables/predictors & $\begin{array}{l}\text { Engel class } \\
\text { I }\end{array}$ & $\begin{array}{l}\text { Engel class } \\
\text { II }\end{array}$ & $\begin{array}{l}\text { Engel class } \\
\text { III }\end{array}$ & $\begin{array}{l}\text { Engel class } \\
\text { IV }\end{array}$ & ${ }^{\star} F, \chi^{2}, \psi$ & df & p Value \\
\hline Age (years) & $37.4( \pm 9.6)$ & $38.9( \pm 9.0)$ & $45.0( \pm 7.5)$ & $28.2( \pm 8.8)$ & 3.34 & $3 / 185$ & 0.020 \\
\hline Duration illness (years) & $23.0( \pm 10.8)$ & $22.1( \pm 11.7)$ & $29.0( \pm 14.7)$ & $20.5( \pm 6.9)$ & 0.89 & $3 / 185$ & 0.444 \\
\hline Age at onset (years) & $14.5( \pm 9.4)$ & $16.8( \pm 6.2)$ & $16.0( \pm 7.9)$ & $7.7( \pm 14.5)$ & 1.17 & $3 / 185$ & 0.324 \\
\hline Gender (male/female ratio) & $83 / 84$ & $6 / 3$ & $6 / 2$ & $1 / 4$ & 0.69 & 1 & 0.406 \\
\hline $\begin{array}{l}\text { Number of seizures prior to epilepsy } \\
\text { surgery }\end{array}$ & $8.5( \pm 13.4)$ & $35.9( \pm 70.7)$ & $26.4( \pm 30.3)$ & $65.6( \pm 124.6)$ & 10.35 & $3 / 185$ & $<0.001$ \\
\hline $\begin{array}{l}\text { Number of seizures after epilepsy } \\
\text { surgery }\end{array}$ & $0.00( \pm 0.00)$ & $1.71( \pm 1.51)$ & $9.0( \pm 9.4)$ & $91.8( \pm 137.8)$ & 33.13 & $3 / 185$ & $<0.001$ \\
\hline $\begin{array}{l}\text { Focal vs focal with secondarily } \\
\text { generalised seizures }\end{array}$ & $160 / 7$ & $9 / 0$ & $8 / 0$ & $4 / 1$ & $-0.01^{*}$ & - & 0.764 \\
\hline TLE vs ETLE & $156 / 11$ & $7 / 2$ & $7 / 1$ & $1 / 4$ & 14.36 & 1 & $<0.001$ \\
\hline Epilepsy location & & & & & See text & & \\
\hline Right & 83 & 6 & 3 & 3 & & & \\
\hline Left & 80 & 3 & 5 & 1 & & & \\
\hline Bilateral & 3 & 0 & 0 & 1 & & & \\
\hline Middle & 1 & 0 & 0 & 0 & & & \\
\hline Lesion & & & & & See text & & \\
\hline Hippocampal sclerosis & 128 & 7 & 5 & 0 & & & \\
\hline Tumour & 28 & 1 & 2 & 2 & & & \\
\hline FCD & 7 & 1 & 0 & 3 & & & \\
\hline AVM & 1 & 0 & 0 & 0 & & & \\
\hline No lesion & 3 & 0 & 1 & 0 & & & \\
\hline Familial history of epilepsia: yes/no & $26 / 141$ & $3 / 6$ & $3 / 5$ & $2 / 3$ & 5.70 & 1 & 0.017 \\
\hline $\begin{array}{l}\text { DD first } 3 \text { months after surgery: yes/ } \\
\text { no }\end{array}$ & $18 / 149$ & $1 / 8$ & $2 / 6$ & $3 / 2$ & 4.77 & 1 & 0.029 \\
\hline Preoperative psychosis: yes/no & $7 / 160$ & $2 / 7$ & $2 / 6$ & $0 / 5$ & $0.19^{*}$ & & 0.026 \\
\hline
\end{tabular}


(and other variables listed in table 2) showed that only dosage of levetiracetam was a significant explanatory variable (Wald $=10.99, \quad \mathrm{df}=1, \quad \mathrm{p}=0.001, \quad$ Nagelkerke= $0.110)$. In the subgroup of patients who had improved at 24 months, the use of levetiracetam showed 1 negative rank, 5 positive ranks and 17 ties, while in those who did not improve there were 16 negative ranks, 5 positive ranks and 166 ties.

Table 2 shows the demographic data of the patients in this study according to Engel's classification. We did not use a $\mathrm{p}$ correction to examine these multiple analyses because these univariate analyses were employed to delineate the possible relevant variables to be used as determinants of independent association with surgery outcome in the ultimate multivariate analyses. There was a marginal but significant difference in age between the Engel classes. Tukey's post hoc test showed that patients in Engel class IV were significantly younger than those belonging to class III $(p=0.011)$. There were no significant differences in duration of illness and age at onset between the Engel classes. The number of presurgery seizures was significantly lower in patients with Engel class I than in those with Engel class II $(p=0.012$ by Tukey's post hoc tests) and class IV $(\mathrm{p}=0.007)$, while there was a trend towards a significant difference with class III $(p=0.068)$. There were no significant differences in number of presurgery seizures between classes II, III and IV. Table 2 shows that the number of postsurgery seizures was significantly different between the four classes. This was validated using the Kruskal-Wallis test $\left(\chi^{2}=150.62, \mathrm{df}=3, \mathrm{p}<0.001\right)$. Tukey's tests showed that all pairwise and post hoc analyses were significant, for example, class I from class II $(\mathrm{p}=0.004)$, class III $(\mathrm{p}<0.001)$ and class IV $(\mathrm{p}<0.001)$, class II from class III $(\mathrm{p}=0.015)$ and class IV $(\mathrm{p}<0.001)$ and class III from class IV $(\mathrm{p}<0.001)$.

Unexpectedly, only a few patients were not allocated to Engel class I, and therefore we were unable to perform $\mathrm{P}^{2}$ tests in the four study groups. Since the major aim of this study is to delineate the characteristics of a good versus a worse surgery outcome, we compared Engel class I versus II+III+IV using $\chi^{2}$ tests or Fisher's exact probability tests. There were no significant associations between Engel class I versus II+III+IV and either gender or focal epilepsy versus focal epilepsy with secondarily generalised seizures. Patients belonging to Engel classes II+III+IV suffered significantly more from ETLE than those belonging to class I. There was no significant difference in right versus left location between Engel class I versus II+III+IV $\left(\chi^{2}=0.29, \mathrm{df}=1, \mathrm{p}=0.591\right)$. There was a weak but significant association between Engel class classification and type of lesion, that is, hippocampal sclerosis (HS) versus other or no lesions $\left(\chi^{2}=4.94, \mathrm{df}=1, \mathrm{p}=0.026\right)$. A positive family history of epilepsy, dysphoric syndrome and preoperative psychosis were significantly associated with Engel classes II+III+IV.

\section{Effects of epilepsy surgery on number of seizures and intake of AEDs}

Table 2 shows the number of seizures before and after epilepsy surgery. RM design ANOVA showed that the number of seizures was significantly reduced by epilepsy surgery $(\mathrm{F}=6.45, \mathrm{df}=1 / 185, \mathrm{p}=0.012)$. The interaction pattern time $\times$ Engel class was significant $(\mathrm{F}=10.34, \mathrm{df}=3$ / $185, \mathrm{p}<0.001)$, showing that epilepsy surgery reduced the number of seizures in classes I, II and III, while in class IV the number of seizures increased further after surgery.

Table 3 shows the differences between postsurgery minus presurgery use of AEDs as binary responses. The discontinuation of AEDs after surgery is shown as negative ranks, the initiation of new AED treatments after surgery as positive ranks, while no changes in the treatments after surgery are shown as ties. McNemar tests for paired data showed that 2 years after epilepsy surgery, phenytoin, carbamazepine, valproic acid, gabapentin, topiramate and clobazam could be discontinued in a significant number of patients, while there were no

Table 3 Effects of epilepsy surgery on the discontinuation of antiepileptic drugs in 189 epilepsy patients

\begin{tabular}{lcccrc}
\hline Drug & - Ranks & + Ranks & Ties & McNemar or Wilcoxon test & Discontinuation rate \\
\hline Phenobarbital & 7 & 1 & 181 & 0.070 & $7 / 37$ \\
Phenytoin & 23 & 3 & 163 & $<0.001$ & $23 / 61$ \\
Carbamazepine & 25 & 1 & 163 & $<0.001$ & $25 / 133$ \\
Valproic acid & 16 & 3 & 170 & 0.004 & $16 / 46$ \\
Clonazepam & 6 & 2 & 181 & 0.289 & $6 / 12$ \\
Gabapentin & 11 & 2 & 176 & 0.022 & $11 / 20$ \\
Lamotrigine & 25 & 4 & 160 & $<0.001$ & $25 / 62$ \\
Topiramate & 13 & 4 & 172 & 0.049 & $13 / 20$ \\
Levetiracetam & 26 & 15 & 148 & 0.118 & $26 / 54$ \\
Clobazam & 29 & 10 & 150 & 0.003 & $29 / 58$ \\
All drugs & 92 & 13 & 84 & $<0.001^{*}$ & - \\
\hline
\end{tabular}

The difference between postsurgery minus presurgery use of antiepileptic drugs is shown as the discontinuation of the drugs after surgery (negative ranks), starting new treatments after surgery (positive ranks) or unchanged treatments after surgery (ties). Thus 'ties' includes patients in whom the specific drugs were not changed and patients who were not using this drug.

${ }^{*}$ All analyses are results of the McNemar test, except the Wilcoxon test $(z=-7.61)$. 
significant changes in the number of patients treated with phenobarbital, clonazepam or levetiracetam. The total number of AEDs was significantly lower after surgery than before surgery. We were able to discontinue one or more AEDs in $48.68 \%$ of the patients, while in $6.88 \%$ of the patients we started a new AED and in $44.44 \%$ of the patients the AED intake was unchanged. RM design ANOVA showed that there was a significant time $\times$ Engel class interaction indicating that the total number of drugs was reduced in class I but not in the other classes. Table 4 shows that the dosages of all AEDs, except clonazepam were lower 24 months after surgery than before.

\section{Prediction of response to epilepsy surgery}

The aforementioned changes in the intake of AEDs after epilepsy surgery suggest that Engel's classification should be adjusted to reflect changes in AED status. Thus, it is clear that there is a difference between patients allocated to, for example, class I and who had their AEDs discontinued/reduced and those belonging to class I but who had an increased/unchanged AED intake. Therefore, we controlled for changes in AED state in two ways: (1) by adjusting statistically for effects of AED state by entering the total number of AEDs prior to and after surgery into the analyses; and (2) by computing a new index of surgery response based on Engel's classification and the AED state. Toward this end, we computed a new score based on Engel classes and the change in AED state from baseline to postsurgery, for example, decreased intake of drugs: rating 1, unchanged: rating 2 and increased: rating 3 . Thus, for class I, this yields three scores, that is, 1 (class I and reduced intake), 2 (class I and unchanged drug state) and 3 (class I but increased drug intake). Applied to all four classes, this method yields a severity score ranging from 1 to 12 . There is a significant association between

Table 4 Effects of epilepsy surgery on the dosage of antiepileptic drugs in 189 patients

\begin{tabular}{llrllr}
\hline & \multicolumn{1}{c}{$\boldsymbol{+}$} & \multicolumn{3}{c}{ Wilcoxon } \\
Drug & Ranks & Ranks & ties & test & p Value \\
\hline Phenobarbital & 12 & 2 & 175 & -2.684 & 0.007 \\
Phenytoin & 43 & 6 & 140 & -4.908 & $<0.001$ \\
Carbamazepine & 65 & 15 & 109 & -6.378 & $<0.001$ \\
Valproic acid & 27 & 7 & 155 & -3.066 & 0.002 \\
Clonazepam & 6 & 3 & 180 & -0.060 & 0.952 \\
Gabapentin & 15 & 5 & 169 & -2.396 & 0.017 \\
Lamotrigine & 42 & 13 & 134 & -4.273 & $<0.001$ \\
Topiramate & 17 & 4 & 168 & -3.047 & 0.002 \\
Levetiracetam & 41 & 22 & 126 & -2.348 & 0.019 \\
Clobazam & 35 & 12 & 141 & -3.219 & $<0.001$ \\
\hline
\end{tabular}

The differences between postsurgery minus presurgery dosages of antiepileptic drugs are given as reduced dosages (negative ranks), increased dosages (positive ranks) or unchanged dosages (ties) after epilepsy surgery.
Table 5 Results of general linear model analysis with the Engel-derived severity score as a dependent variable and the listed variables as predictor variables

\begin{tabular}{lrrr}
\hline Explanatory variables & F & df & p Value \\
\hline TLE vs ETLE & 16.04 & 1 & $<0.001$ \\
No family history of epilepsy & 5.19 & 1 & 0.024 \\
Number of seizures presurgery & 11.45 & 1 & $<0.001$ \\
Dysphoric disorder within the first & 6.29 & 1 & 0.013 \\
3 months after surgery & & & \\
Use of gabapentin postsurgery & 7.04 & 1 & 0.009 \\
\hline ETLE, extratemporal lobe epilepsy; TLE, temporal lobe epilepsy.
\end{tabular}

Engel's class classification and this newly presented severity score (Spearman's correlation: $r=0.592$, $\mathrm{p}<0.001)$.

Table 5 shows the outcome of a general linear model analysis with this new severity score as a dependent variable and the variables listed in table 2 and the drug state of the patients as predictor variables. Up to $25.2 \%$ in the variance of the severity index was explained by five variables $(\mathrm{F}=12.32, \mathrm{df}=5 / 183, \mathrm{p}<0.001)$ : a lower severity score was associated with TLE versus ETLE and a negative family history of epilepsy; a worse outcome was predicted by an increased number of seizures before surgery, dysphoric syndrome the first 3 months after surgery and use of gabapentin. Using a threshold value $>9$ for the total number of seizures before surgery showed a similar significant effect $(\mathrm{F}=8.99, \quad \mathrm{df}=1$, $\mathrm{p}=0.003$ ), suggesting that a threshold value of 9 or more may be used as a predictor variable. Entering the use of AEDs, total number of AEDs and dosages of AEDs before and after surgery (at 24 months) as explanatory variables showed that none of these variables, except gabapentin postsurgery, was significant in explaining the severity index and that entering these drug variables did not change the results.

We have also examined the prediction of the Engel classes using the same variables as in table 5 but considering that the Engel classes are continuous classes or ordinal variables ranging from one (for class I) to four (for class IV). Generalised linear model analysis showed that this Engel scaling was predicted by five variables: the outcome was better when suffering from TLE (Wald=20.33, $\mathrm{df}=1, \mathrm{p}<0.001$ ) and having a negative family history (Wald $=9.21, \mathrm{df}=1, \mathrm{p}=0.002$ ), while the total number of presurgery seizures (Wald $=17.03, \mathrm{df}=1$, $\mathrm{p}<0.001$ ), dysphoric syndrome (Wald $=7.91, \quad \mathrm{df}=1$, $\mathrm{p}=0.005$ ) and use of gabapentin (Wald $=8.19$, $\mathrm{df}=1$, $\mathrm{p}=0.004$ ) predicted a worse outcome.

Table 6 shows the results of an automatic stepwise logistic regression analysis with Engel class I as a dependent variable (classes III+IV as a reference group) and the variables listed in table 2 (and number of baseline epileptic seizures $>9$, yes or no) as predictors. We found that five variables were significantly associated with Engel class I $\left(\chi^{2}=31.88, \quad \mathrm{df}=5, \mathrm{p}<0.001\right.$, Nagelkerke $=0.401$; correctly 
Table 6 Results of logistic regression with Engel class I as a dependent variable and the listed variables as predictors

\begin{tabular}{lrrrrrr}
\hline Predictors & Wald & df & p Value & \multicolumn{1}{c}{ OR } & 95\% Cl, lower & 95\% Cl, upper \\
\hline TLE vs ETLE & 12.77 & 1 & $<0.001$ & 20.52 & 3.91 & 107.60 \\
Negative family history of epilepsia & 5.24 & 1 & 0.024 & 5.72 & 1.28 & 25.47 \\
Less than 9 seizures before epilepsy surgery & 6.40 & 1 & 0.011 & 6.64 & 1.53 & 28.77 \\
Dysphoric disorder first 3 months after surgery & 4.19 & 1 & 0.041 & 0.19 & 0.041 & 0.93 \\
Age & 4.90 & 1 & 0.027 & 1.09 & 1.01 & 1.18 \\
\hline
\end{tabular}

ETLE, extratemporal lobe epilepsy; TLE, temporal lobe epilepsy.

classified cases $=92.8 \%$, namely TLE versus ETLE, a negative family history of epilepsy, less than nine seizures before surgery, age and the presence of dysphoric syndrome. These associations remained significant after adjusting (forced entry) for the effects of epilepsy location, type of lesion and focal epilepsy with or without secondarily generalised seizures in logistic regression analyses.

\section{DISCUSSION}

A first major finding of this study is that there were significant differences in surgery outcome between 6 and 24 months after surgery: our success rate at 6 months $(78.8 \%)$ had increased significantly $(88.3 \%)$ at 24 months. We have performed post hoc analyses to examine the characteristics of the group of patients who had improved at 24 months. We found that there was only one significant, although weak, predictor, that is, use of levetiracetam. Thus, in a few patients, the increased use of levetiracetam postsurgery may be associated with a better outcome at 24 months, whereas in most of the patients no specific characteristics were detected. Already in 1970, it was suggested that some patients may show seizures after surgery that eventually remit some months to years later, that is, the 'running down phenomenon'. 13 Nevertheless, our findings contradict one of the largest series of epilepsy surgery results, showing a gradual decline over time in the estimated proportion of patients who remain seizure free. ${ }^{13}$ In another study, it was reported that the prevalence of Engel class I was $76.2 \%$ at 6 months, $72.3 \%$ at 2 years and $71.1 \%$ at 5 years. ${ }^{14}$ The prevalence of being completely seizure free at 12 and 18 years after mesial TLE/ HS surgery was $65 \%$ and $62 \%$, respectively. ${ }^{15} \mathrm{~A}$ meta-analysis showed seizure control to decline over time, especially after 2 years. ${ }^{16}$ The risk of having any recurrence was $22 \%$ during the first 24 months and it increased $1.4 \%$ per year afterwards. ${ }^{15}$ Some of the longterm postsurgical follow-up studies supported the concept that the prognosis may improve over time, for example, less memory decline. ${ }^{17}$ One explanation of these contradictory data is that a running down phenomenon may occur in an initially non-equilibrium period, with an undetermined duration, and that seizures may reoccur after that time point.
The high success rate of epilepsy surgery in our hospital (ie, $88.3 \%$ at 24 months) may be explained by our inclusion criteria, which are based on clinical semiology, $24 \mathrm{~h} \mathrm{EEG}$ and MRI, to identify patients with primary epileptogenic lesions. As a consequence, this cohort comprises only $2 \%$ MRI-negative epilepsy and $>90 \%$ temporal lobe surgery patients. Therefore, our findings may be more difficult to readily extrapolate to more heterogeneous cohorts including high rates of non-lesional and/or extratemporal epilepsy. Nevertheless, the associations of Engel class outcome with the significant predictors (discussed in detail below) were not affected after adjusting for the effects of epilepsy type and aetiology in the multivariate analyses.

A second major finding is that the efficacy of epilepsy surgery was reflected not only by the reduced number of seizures, but also by the reduced use of AEDs. We were able to discontinue one or more AEDs in $48.7 \%$ of the patients, while $13.2 \%$ of all patients were free of any AEDs 2 years after surgery. This discontinuation rate might be slightly lower than that in previous reports which showed that around $52.6 \%$ of the patients can discontinue AEDs at 2 years without seizure recurrence. ${ }^{18}$ In another study, $28.1 \%$ of the patients had discontinued AED treatment 2 years after surgery and had remained seizure free, suggesting that there was no risk of seizure recurrence after discontinuation of AEDs. ${ }^{14} \mathrm{~A}$ meta-analysis showed that in patients with all types of surgery, 20\% achieved long-term AED discontinuation, $31 \%$ remained on polytherapy and $41 \%$ were on monotherapy. ${ }^{19}$ In addition, we found no significant associations between AED discontinuation and seizure freedom. Other studies report that AED discontinuation may be a strong predictor for seizure recurrence in postsurgery seizure-free cases. ${ }^{12}$ Boshuisen et $a l^{20}$ in a study performed on children with intractable epilepsy, found that AED withdrawal did not affect long-term seizure outcome but may unmask incomplete surgical success sooner, identifying children who need continuous drug treatment. One of our analyses showed that use of gabapentin was a significant predictor variable for a worse outcome. In our clinic, however, gabapentin was not the first AED choice for seizure treatment and was used in refractory seizures that failed to respond to treatment with other AEDs. This may show that use of gabapentin should not be regarded as a real explanatory variable 
but as a post hoc adjustment for possible effects of the drug state.

The third major finding of this study is that a good outcome of epilepsy surgery, that is, being allocated to class I, could be predicted by TLE versus ETLE, less than nine presurgery seizures per month, a negative familial history of epilepsy, age and absence of a dysphoric disorder. TLE was the most significant outcome predictor. This finding is consistent with most published papers showing that TLE, in the short-term and longterm monitoring periods ${ }^{21}$ and in paediatric and adult patients, has a significantly better postoperative outcome than ETLE. ${ }^{22}$ In paediatric patients, the seizure-free rate in TLE was $71.8 \%$ vs $59.7 \%$ in ETLE, whereas in adult epilepsy the seizure-free rate in TLE was $69.4 \%$ vs $45.9 \%$ in ETLE. ${ }^{22}$ In ETLE, it is more difficult to localise the epileptogenic focus to a specific cerebral region and to completely remove the epileptogenic region without impairing the eloquent cortex. ${ }^{23}$

The second predictor, that is, number of preoperative seizures, shows that surgery may not be the best treatment option for patients with many refractory seizures. A high number of presurgery seizures might indicate multiple types of seizures, unidentified multiple lesions or severe pathology or other factors negatively modifying the surgery outcome. We established that a threshold value of 9 seizures/month best predicted Engel class I membership, while another study delineated that more than 30 seizures/month best predicted a negative outcome. ${ }^{1}$ These differences between both studies may reflect differences in sensitivity and specificity. Thus, we established that $<9$ seizures/month significantly predicts Engel class I versus II+III+IV, while it is obvious that if we had used Engel class I+II+III versus IV, the threshold value would have been higher.

To the best of our knowledge, this is the first study to show that a positive family history for epilepsy may worsen postsurgery outcome. There is now evidence that the risk to develop epilepsy is significantly increased in the first-degree relatives of people with epilepsy of unknown cause. ${ }^{24}$ Twin studies consistently show higher concordance in monozygotic than in dizygotic pairs. ${ }^{25}$ However, the genes identified so far affect risk in a very small proportion of patients, while most epilepsies occur in the absence of a significant family history. ${ }^{26}$ In a few clinical studies on epilepsy with or without psychiatric disorders, genetic linkage is considered to increase risk of poor clinical outcome. ${ }^{27} 28$ Thus, a positive family history of epilepsy occurs more frequently in TLE with postictal psychosis than in TLE alone. ${ }^{27}$ A positive family history of epilepsy also has a significantly negative impact on the quality of life. ${ }^{28}$ The inverse relationship between a family history of epilepsy and Engel class outcome may be explained by mutations in specific genes that are related to a more severe outcome, including drug resistance, and distinct neuroradiological findings as have been observed in benign neonatal epilepsy or benign familial neonatal convulsions. ${ }^{29}{ }^{30}$ However, it is unlikely that single common variants could explain more than $4.4 \%$ of outcome variation in newly treated epilepsy. ${ }^{31}$ Therefore, it should be examined whether multiple common variants may underpin increased resistance to resective epilepsy surgery.

There are only few studies in adults that have examined age as a predictor of surgery outcome. In individuals aged less than 50 years, $58 \%$ were allocated to Engel class I, while $74 \%$ of those who were aged more than 50 years and $91 \%$ of those who were aged more than 60 years were allocated to Engel class I. ${ }^{16}$ There were no differences in surgical outcome in terms of medication withdrawn between older and younger patients (threshold value $=50$ years), but more surgical complications in the older group. ${ }^{32}$ Previous studies in children or teenagers showed that early surgical treatment correlated with a better Engel class outcome. ${ }^{33} 34$ In our study, however, the absolute differences in age were very small. Therefore, further research should delineate whether age at surgery contributes significantly to Engel class outcome.

The fifth predictor of surgery outcome is dysphoric disorder, that is, labile and irritable mood emerging within 3 months after surgery, but most often the first 12 months. While presurgery psychiatric factors are identified as predictors of a worse surgery outcome, few studies have examined postsurgery psychiatric predictors. Epilepsy is accompanied by the interictal dysphoric disorder, characterised by intermittent affective symptoms including labile affective symptoms, paroxysmal irritability and outbursts of aggressive behaviour. ${ }^{35} 36$ The prevalence of interictal dysphoric disorder (and having no depression and dysthymia according to the MINI) is around $48.2 \%$ in patients with epilepsy. ${ }^{36} 37$ There is some evidence suggesting that interictal dysphoric disorder and peri-ictal dysphoric syndrome may be separate syndromes. ${ }^{37}$ Therefore, it may be hypothesised that dysphoric disorder in the early postoperative period is in fact interictal dysphoric disorder and that in those patients subsyndromal seizure activity is present despite surgery, thereby predicting future clinical seizures. Future research should delineate this symptom complex in association with surgery outcome using the 38-item Interictal Dysphoric Disorder Inventory (IDDI) ${ }^{37}$ Emotional reactivity is a psychosocial stressor increasing circulating glucocorticoid levels causing an increased vulnerability to amygdala kindling. ${ }^{38}$ Kindling is the process by which repeated minor stimulations (electrical or chemical) of the brain are associated with epileptogenesis and the onset of mood disturbances. ${ }^{39}$

The limitations of this study are the shorter follow-up period (24 months) and the lower number of patients not allocated to Engel class I as a result of the unexpectedly high success rate of epilepsy surgery in this cohort. As such, the results should be interpreted with caution. Future research should validate the predictors delineated in our study and use the IDDI to score severity of the dysphoric syndrome. 
Acknowledgements The authors would like to thank Dr Chaichon Loechareonkul, the former director of the Comprehensive Epilepsy Unit, for his help and encouragement

Contributors BK made the study design. BK, CL, TS and MM interpreted the data. BK and MM performed the statistical analyses and wrote the manuscript. CL, TS and BK collected the data.

Funding This research received no specific grant from any funding agency in the public, commercial or not-for-profit sectors.

Competing interests None.

Patient consent Obtained.

Ethics approval The study was approved by the Ethics Research Committee/ Institutional Review Board (IRB) at Chulalongkorn University, Department of Medicine, Bangkok, Thailand.

Provenance and peer review Not commissioned; externally peer reviewed.

Data sharing statement No additional data are available.

Open Access This is an Open Access article distributed in accordance with the Creative Commons Attribution Non Commercial (CC BY-NC 4.0) license, which permits others to distribute, remix, adapt, build upon this work noncommercially, and license their derivative works on different terms, provided the original work is properly cited and the use is non-commercial. See: http:// creativecommons.org/licenses/by-nc/4.0/

\section{REFERENCES}

1. Edelvik A, Rydenhag B, Olsson I, et al. Long-term outcomes of epilepsy surgery in Sweden: a national prospective and longitudinal study. Neurology 2013;81:1244-51.

2. Sarkis RA, Jehi L, Najm IM, et al. Seizure outcomes following multilobar epilepsy surgery. Epilepsia 2012;53:44-50.

3. Kanchanatawan B, Kasalak R. Quality of life in Thai intractable epileptic patients with and without surgery. J Med Assoc Thai 2012;95:1232-8.

4. Rasmussen T. The neurosurgical treatment of epilepsy. In: Niedermeyer E, ed. Epilepsy: modern problems of pharmacopsychiatry. Basel: Karger, 1970:306-25.

5. Janszky J, Janszky I, Schulz R, et al. Temporal lobe epilepsy with hippocampal sclerosis: predictors for long-term surgical outcome. Brain 2005;128(Pt 2):395-404.

6. Cleary RA, Thompson PJ, Fox Z, et al. Predictors of psychiatric and seizure outcome following temporal lobe epilepsy surgery. Epilepsia 2012;53:1705-12.

7. Teutonico F, Mai R, Devinsky O, et al. Epilepsy surgery in tuberous sclerosis complex: early predictive elements and outcome. Childs Nerv Syst 2008;24:1437-45.

8. Kanner AM, Byrne R, Chicharro A, et al. A lifetime psychiatric history predicts a worse seizure outcome following temporal lobectomy. Neurology 2009;72:793-9.

9. Guarnieri R, Walz R, Hallak JE, et al. Do psychiatric comorbidities predict postoperative seizure outcome in temporal lobe epilepsy surgery? Epilepsy Behav 2009;14:529-34.

10. Kanner AM. Do psychiatric comorbidities have a negative impact on the course and treatment of seizure disorders? Curr Opin Neurol 2013;26:208-13.

11. Adams SJ, Velakoulis D, Kaye $\mathrm{AH}$, et al. Psychiatric history does not predict seizure outcome following temporal lobectomy for mesial temporal sclerosis. Epilepsia 2012;53:1700-4.

12. Pimentel J, Peralta AR, Campos A, et al. Antiepileptic drugs management and long-term seizure outcome in post surgical mesial temporal lobe epilepsy with hippocampal sclerosis. Epilepsy Res 2012;100:55-8.

13. de Tisi J, Bell GS, Peacock JL, et al. The long-term outcome of adult epilepsy surgery, patterns of seizure remission, and relapse: a cohort study. Lancet 2011;378:1388-95.

14. Elsharkawy AE, May T, Thorbecke R, et al. Long-term outcome and determinants of quality of life after temporal lobe epilepsy surgery in adults. Epilepsy Res 2009;86:191-9.

15. Hemb M, Palmini A, Paglioli E, et al. An 18-year follow-up of seizure outcome after surgery for temporal lobe epilepsy and hippocampal sclerosis. J Neurol Neurosurg Psychiatry 2013;84:800-5.
16. Patra S, Elisevich K, Podell K, et al. Influence of age and location of ictal onset on postoperative outcome in patients with localization-related epilepsy. Br J Neurosurg 2014;28:61-7.

17. Andersson-Roswall L, Malmgren K, Engman E, et al. Verbal memory decline is less frequent at 10 years than at 2 years after temporal lobe surgery for epilepsy. Epilepsy Behav 2012;24:462-7.

18. Rathore C, Panda S, Sarma PS, et al. How safe is it to withdraw antiepileptic drugs following successful surgery for mesial temporal lobe epilepsy? Epilepsia 2011;52:627-35.

19. Téllez-Zenteno JF, Dhar R, Hernandez-Ronquillo $L$, et al. Long-term outcomes in epilepsy surgery: antiepileptic drugs, mortality, cognitive and psychosocial aspects. Brain 2007;130(Pt 2):334-45.

20. Boshuisen $\mathrm{K}$, Arzimanoglou $\mathrm{A}$, Cross $\mathrm{JH}$, et al. Timing of antiepileptic drug withdrawal and long-term seizure outcome after paediatric epilepsy surgery (TimeToStop): a retrospective observational study. Lancet Neurol 2012;11:784-91.

21. Mohammed HS, Kaufman CB, Limbrick DD, et al. Impact of epilepsy surgery on seizure control and quality of life: a 26-year follow-up study. Epilepsia 2012;53:712-20.

22. $\mathrm{Yu}$ T, Zhang G, Kohrman MH, et al. A retrospective study comparing preoperative evaluations and postoperative outcomes in paediatric and adult patients undergoing surgical resection for refractory epilepsy. Seizure 2012;21:444-9.

23. Ansari SF, Tubbs RS, Terry CL, et al. Surgery for extratemporal nonlesional epilepsy in adults: an outcome meta-analysis. Acta Neurochir 2010;152:1299-305.

24. Ottman R, Annegers JF, Risch N, et al. Relations of genetic and environmental factors in the etiology of epilepsy. Ann Neurol 1996;39:442-9.

25. Berkovic SF, Howell RA, Hay DA, et al. Epilepsies in twins: genetics of the major epilepsy syndromes. Ann Neurol 1998;43:435-45.

26. Ottman R, Risch N. Genetic epidemiology and gene discovery in epilepsy. In: Noebels JL, Avoli M, Rogawski MA, Olsen RW, Delgado-Escueta AV, eds. Jasper's basic mechanisms of the epilepsies. 4th edn. Bethesda, MD: National Center for Biotechnology Information, 2012.

27. Cleary RA, Thompson PJ, Thom M, et al. Postictal psychosis in temporal lobe epilepsy: risk factors and postsurgical outcome? Epilepsy Res 2013;106:264-72.

28. Pauli C, Thais ME, Claudino LS, et al. Predictors of quality of life in patients with refractory mesial temporal lobe epilepsy. Epilepsy Behav 2012;25:208-13.

29. Soldovieri MV, Boutry-Kryza N, Milh M, et al. Novel KCNQ2 and KCNQ3 mutations in a large cohort of families with benign neonatal epilepsy: first evidence for an altered channel regulation by syntaxin-1A. Hum Mutat 2014;35:356-67.

30. Borgatti R, Zucca C, Cavallini A, et al. A novel mutation in KCNQ2 associated with BFNC, drug resistant epilepsy, and mental retardation. Neurology 2004;63:57-65.

31. Speed D, Hoggart C, Petrovski S, et al. A genome-wide association study and biological pathway analysis of epilepsy prognosis in a prospective cohort of newly treated epilepsy. Hum Mol Genet 2014:23:247-58.

32. Srikijvilaikul T, Lerdlum S, Tepmongkol S, et al. Outcome of temporal lobectomy for hippocampal sclerosis in older patients. Seizure 2011;20:276-9.

33. Jo KI, Shin HJ, Hong SC. Seizure outcomes of lesionectomy in pediatric lesional epilepsy with brain tumor-single institute experience. Brain Dev 2013;35:810-15.

34. Simasathien T, Vadera S, Najm I, et al. Improved outcomes with earlier surgery for intractable frontal lobe epilepsy. Ann Neurol 2013;73:646-54.

35. Blumer D, Montouris G, Davies K. The interictal dysphoric disorder: recognition, pathogenesis, and treatment of the major psychiatric disorder of epilepsy. Epilepsy Behav 2004;5:826-40.

36. Mula M, Jauch R, Cavanna A, et al. Clinical and psychopathological definition of the interictal dysphoric disorder of epilepsy. Epilepsia 2008;49:650-6.

37. Mula M, Jauch R, Cavanna A, et al. Interictal dysphoric disorder and periictal dysphoric symptoms in patients with epilepsy. Epilepsia 2010;51:1139-45.

38. Jones NC, Lee HE, Yang M, et al. Repeatedly stressed rats have enhanced vulnerability to amygdala kindling epileptogenesis. Psychoneuroendocrinology 2013;38:263-70.

39. Post RM. Kindling and sensitization as models for affective episode recurrence, cyclicity, and tolerance phenomena. Neurosci Biobehav Rev 2007;31:858-73. 